\title{
Light-emitting Diodes for Manipulating the Phytochrome Apparatus
}

\author{
Gary W. Stutte \\ Dynamac Corporation, Space Life Sciences Laboratory, Mail Code DYN-3, Kennedy Space Center, FL 32899
}

Additional index words. LEDs, photostationary state, strawberry, lettuce, Lactuca sativa, Fragaria $\times$ ananassa

\begin{abstract}
Light-emitting diodes (LEDs) are solid-state, long-lived, durable sources of narrow-band light output that can be used in a range of horticultural and photobiological applications. LED technology is rapidly developing and high-quality, high-output LEDs are becoming commercially available at an affordable cost. LEDs provide the opportunity to optimize the spectra for a given plant response, but consideration must be given to both photosynthetic and photomorphogenic effects of light while making those selections. A discussion of basic phytochrome response and data necessary to select narrow-band LEDs to achieve a specific photostationary state is provided. The use of LEDs to alter spectral quality, and phytochrome equilibrium, to regulate anthocyanin formation in red leaf lettuce and to regulate flowering of short-day strawberry are discussed.
\end{abstract}

Light-emitting diodes (LEDs) are solidstate semiconductor devices that produce narrow spectrum light when voltage is applied. The use of LEDs in photobiology research was reported in the 1980s and the concept of using LEDs for plant lighting was evaluated for space flight applications in the 1990s. LED technology has developed rapidly over the past decade with increasing flux output becoming available at decreasing prices. As the price of LEDs has decreased, there has been a renewed interest in the use of LEDs as tools for photobiology as well (Folta and Childers, 2008). With the increasing availability of LED light sources at affordable prices, the range of commercial applications has also been expanding; commercial lettuce production facilities using LED light sources have been built in Japan (Wheeler, 2008), light fixtures for intracanopy lighting are being developed (Massa et al., 2008), and supplemental lighting fixtures have been designed (Bourget, 2008). Although a fundamental shift in lighting technologies for controlled environments has great promise, there are a number of significant issues associated with photobiology that need to be considered with using narrow-spectrum LEDs in crop production.

As the price of LEDs decline, and the available wavelengths increase, there is increasing opportunity to incorporate LEDs into commercial horticultural practice. To efficiently implement LEDs into commercial horticulture production practices, it is necessary to match the particular spectral characteristics of the light source with the photosynthetic and photomorphogenic requirements of the crop of interest.

The objectives of this article are: 1) to discuss characteristics and availability of LEDs for plant research; 2) to provide a brief review of phytochrome physiology; 3) to discuss basic concepts in selecting LEDs for horticulture; and 4) to discuss two examples

\footnotetext{
Received for publication 23 Oct. 2008. Accepted for publication 30 Nov. 2008.

${ }^{1}$ To whom reprint requests should be addressed; e-mail gary.w.stutte@nasa.gov.
}

from the horticultural literature of how LEDs have been used to alter crop development.

\section{BACKGROUND ON LIGHT- EMITTING DIODES}

LEDs are solid-state, durable, long-lived light sources that provide narrow-band spectral emissions. Light from an LED is generated inside the $p$-n junction of a simple diode, which is made of two different alloys of different potential energies. As electrical current moves from one alloy to an alloy of a lower potential energy, photons are released. This potential difference, known as the energy gap, determines the wavelength of the photons produced. The composition of the different materials changes the energy gap and thus changes the color of light that is generated. The first infrared LED was patented in 1961 and the first practical visible spectra LED patented in 1962. There was rapid development of the technology in the 1980s and the first high-output red LEDs became available in the early 1990s (Bourget, 2008, and references therein). NASA became involved with the selection and development of LED light sources for plant growth in the mid-1990s (Goins et al., 1997; Kim et al., 2005; Morrow, 2008). NASA support resulted in a number of specialized lighting systems for the Space Shuttle and the International Space Station and provided groundwork for development of many other horticultural applications (Kim et al., 2005; Morrow, 2008).

LEDs have several unique characteristics that make them desirable for use in controlled environment research, as tools for photobiology, and, increasingly, as supplemental lighting for greenhouse systems. These include the ability to control spectral composition, provide high light output with low radiant heat, flexibility in design and placement, and exceptionally long life cycles between failures (Morrow, 2008). LEDs are becoming available in an increasing number of wavelengths (Bourget, 2008). Table 1 provides a list of commercially available LEDs that may be of interest to horticulturists and indicates the general color, the wavelengths that are available within that range, and the probable composition of the chip alloy. Table 1 is not meant to be exhaustive, but does indicate that the opportunities to precisely define spectra for specific needs and applications will be available in the foreseeable future.

\section{PHYTOCHROME}

Phytochrome, and its central role in photoregulation of plant development, has been the subject of extensive study for over 60 years (Borthwick et al., 1954; Franklin and Whitelam, 2005; Smith and Whitelam, 1990); and many excellent reviews of its discovery (Borthwick, 1972), chemistry (Pratt, 1982), structure (Rockwell et al., 2006), mode of action (Bae and Choi, 2008; Chen et al., 2004), and molecular biology (Paul and Khurana, 2008) have been published and are not considered here.

Phytochromes are a family of proteins that have two forms, the red absorbing form $\left(\mathrm{P}_{\mathrm{r}}\right)$ and the far red absorbing form $\left(\mathrm{P}_{\mathrm{fr}}\right)$. The $\mathrm{P}_{\mathrm{r}}$ form, which has $\lambda_{\max }=660 \mathrm{~nm}$, undergoes a conformational shift to the $\mathrm{P}_{\mathrm{fr}}$ form when it absorbs light. The $\mathrm{P}_{\mathrm{fr}}$ form, which has $\lambda_{\max }=$ $730 \mathrm{~nm}$, undergoes a conformational shift to the $\mathrm{P}_{\mathrm{r}}$ form when it absorbs light. The $\mathrm{P}_{\mathrm{fr}}$ form is generally considered to be the active form (Smith and Whitelam, 1990). This generalized response is shown schematically in Figure 1.

When investigating phytochrome responses, it would at first appear that an investigator should be very concerned about the relative balance between the $\lambda=660 \mathrm{~nm}$ and $\lambda=730 \mathrm{~nm}$ in the light source. Although important in selecting a lamp source, in practice, the most relevant factor in photobiology is the fraction of phytochrome present in the active $\left(\mathrm{P}_{\mathrm{fr}}\right)$ form with respect to the total phytochrome $\left(\mathrm{P}_{\text {tot }}=\mathrm{P}_{\mathrm{fr}}+\mathrm{P}_{\mathrm{r}}\right)$ at photoequilibrium.

Although $\mathrm{P}_{\mathrm{r}}$ has $\lambda_{\max }=660 \mathrm{~nm}$ and $\mathrm{P}_{\mathrm{fr}}$ at $\lambda_{\text {max }}=730 \mathrm{~nm}$, there is significant overlap in the relative spectral absorbance of $\mathrm{P}_{\mathrm{r}}$ and $\mathrm{P}_{\mathrm{fr}}$ (Fig. 2). As a result of the relative differences in absorption and the subsequent conformational change between $\mathrm{P}_{\mathrm{r}}$ and $\mathrm{P}_{\text {fr }}$, both forms are present in the plant. The relative proportion 
Table 1. Color designation, wavelength $(\lambda)$ range, available wavelengths, and semiconductor material of commercially available light-emitting diodes (LEDs) from the ultraviolet $(\lambda<400 \mathrm{~nm})$ to near infrared $(\lambda>760 \mathrm{~nm})^{\mathrm{z}}$

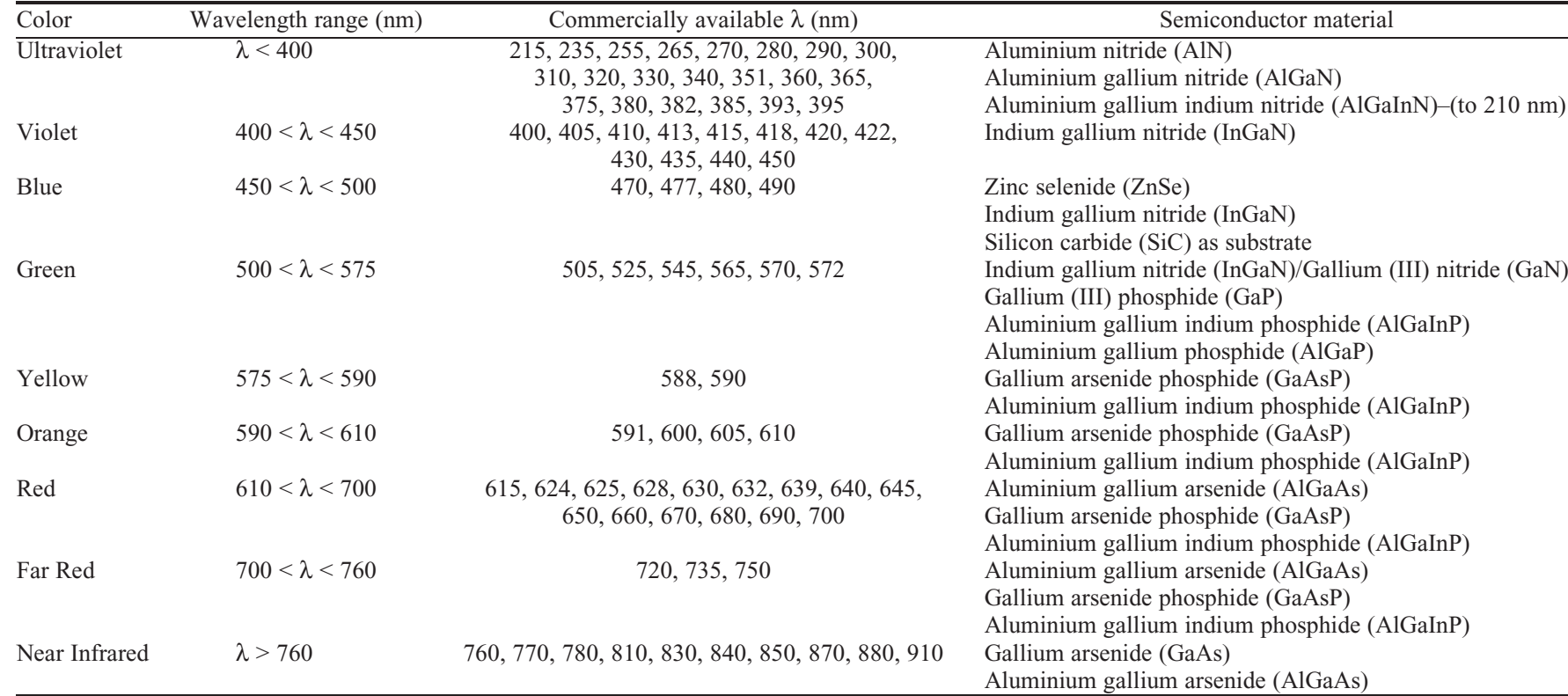

${ }^{2}$ Peak wavelength data and semiconductor material were obtained from technical specifications provided by the manufacturer(s) and have not been independently verified by the author. The listing of availability of a particular LED does not imply that it has the output or operational parameters to meet a particular application. The listing is provided for guidance only and should not be used to develop experimental protocols without confirming that output will meet the user requirements.

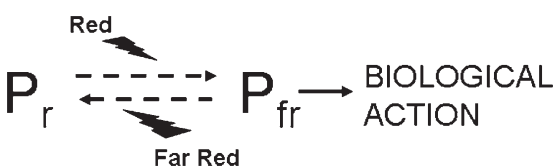

Fig. 1. Schematic of generalized response of phytochrome forms $\mathrm{P}_{\mathrm{r}}$ and $\mathrm{P}_{\mathrm{fr}}$ to red $\left(\lambda_{\max }=\right.$ $660)$ and far red $\left(\lambda_{\max }=730\right)$ radiation.

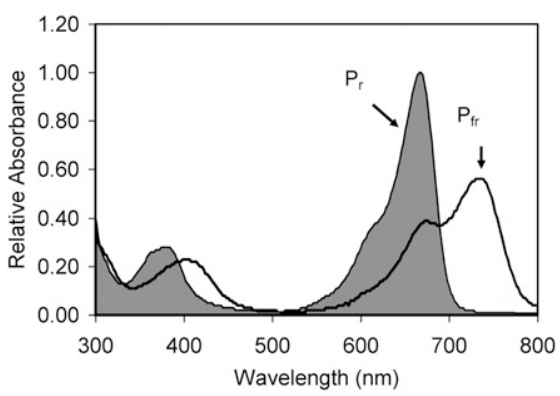

Fig. 2. Relative absorption spectra of $\mathrm{P}_{\mathrm{r}}$ and $\mathrm{P}_{\mathrm{fr}}$ forms of phytochrome for wavelengths ranging from 300 to $800 \mathrm{~nm}$. (Data derived from Sager et al., 1988.)

of active form $\left(\mathrm{P}_{\mathrm{fr}}\right)$ to the total $\left(\mathrm{P}_{\text {tot }}\right)$ is considered the phytochrome photostationary state $(\Phi)$. It is this relative proportion of $\mathrm{P}_{\mathrm{fr}}$ to $\mathrm{P}_{\text {tot }}$ that regulates a given photomorphogenic response. Because the absorption spectrum of each form is known, it is possible to estimate $\Phi$ if the spectral distribution of a light source is also known (Sager and McFarlane, 1997).

The phytochrome photostationary state $(\Phi)$ is established by multiplying the irradiance $(N)$ at each wavelength $(\lambda)$ against the relative absorption at that $\lambda$ for each form

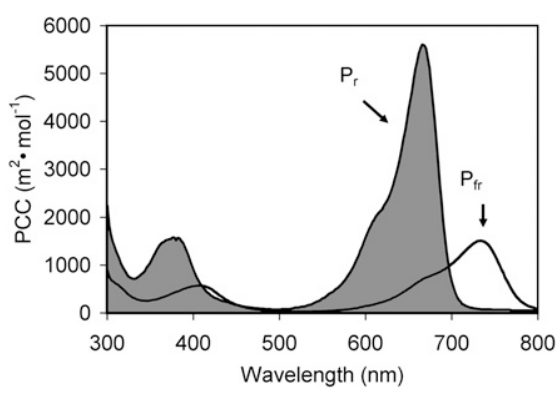

Fig. 3. Phytochrome cross-sectional (PCC) area of $\mathrm{P}_{\mathrm{r}}$ and $\mathrm{P}_{\mathrm{fr}}$ forms of phytochrome for wavelengths ranging from 300 to $800 \mathrm{~nm}$. (Data derived from Sager and McFarlane, 1997.)

of phytochrome [the photochemical crosssectional area $(\sigma)$ ] (Fig. 3) and calculating $\Phi$ using the following equation:

$\Phi=\left[\sum_{800}^{800} N_{\lambda} \sigma_{r_{\lambda}}\right] \div\left[\sum_{800}^{800} N_{\lambda} \sigma_{r_{\lambda}}=\sum_{800}^{800} N_{\lambda} \sigma_{f r_{\lambda}}\right]$.

Typical values of $\Phi$ under ambient solar conditions are $\Phi=0.6$ for full sun and $\Phi=0.1$ for dense shade under a full canopy (Salisbury and Ross, 1992; Nagy and Schaefer, 2002), although these values vary according to canopy type and density (Vandenbussche et al., 2005). The range of values from electric light sources vary from $\Phi=0.1$ from a far red rich light source to $\Phi=0.89$ from a source with high red spectrum (Sager and McFarlane, 1997).

The values for estimating $\Phi$ derived from isolated phytochrome (Sager and McFarlane, 1997; Sager et al., 1988) are useful guides to determining the effect of any light source on the phytochrome response. When using narrow-band LEDs, the $\Phi$ can be approximated based on the $\lambda_{\max }$ of the LED. Table 2 shows the estimate of $\Phi$ for discrete narrow-band LEDs with $\lambda_{\max }$ from 300 to $800 \mathrm{~nm}$. Table 2 also includes relative quantum efficiency (RQE) for photosynthesis (McCree, 1972) to allow the photosynthetic efficiency of a given wavelength to be evaluated as well. These well-defined parameters allow the spectra to be optimized for both photosynthesis and photomorphogenesis.

\section{CONSIDERATIONS IN SELECTING LIGHT-EMITTING DIODES FOR PLANT RESEARCH}

The increasing availability of LEDs provides the investigator the opportunity to optimize wavelength selection for photomorphogenic as well as photosynthetic responses (Fig. 4). The selection of appropriate wavelengths to optimize photosynthesis, for example $440 \mathrm{~nm}$ and $660 \mathrm{~nm}$, can be supplemented with wavelengths to alter the photo stationary state.

By selecting wavelengths with high RQE and appropriate $\Phi$, desired growth and morphological characteristics can be achieved. Although the spectral quality is of primary concern in the selection of appropriate LED light sources, a number of other issues should also be considered. These include spatial distribution and total output. LEDs are inherently point source producers of light, which is emitted in a Lambertian manner. As such, the design of luminare to achieve light distribution is critical for many applications. 
Table 2. Spectral files of relative quantum efficiency $(\mathrm{RQE})^{z}$ and phytochrome phytostationary state (PSS) ${ }^{y}$ at $10 \mathrm{~nm}$ wavelength intervals from 300 to $800 \mathrm{~nm}^{\mathrm{x}}$

\begin{tabular}{|c|c|c|c|c|c|}
\hline Wavelength (nm) & RQE & PSS & Wavelength (nm) & RQE & PSS \\
\hline 310 & 0.00 & 0.68 & 560 & 0.91 & 0.90 \\
\hline 320 & 0.01 & 0.66 & 570 & 0.94 & 0.90 \\
\hline 330 & 0.04 & 0.69 & 580 & 0.96 & 0.91 \\
\hline 340 & 0.10 & 0.76 & 590 & 0.99 & 0.92 \\
\hline 350 & 0.16 & 0.80 & 600 & 1.00 & 0.92 \\
\hline 360 & 0.27 & 0.82 & 610 & 1.00 & 0.92 \\
\hline 370 & 0.40 & 0.81 & 620 & 1.00 & 0.90 \\
\hline 380 & 0.50 & 0.77 & 630 & 0.99 & 0.89 \\
\hline 390 & 0.59 & 0.73 & 640 & 0.96 & 0.89 \\
\hline 400 & 0.66 & 0.62 & 650 & 0.94 & 0.89 \\
\hline 410 & 0.71 & 0.50 & 660 & 0.93 & 0.89 \\
\hline 420 & 0.75 & 0.42 & 670 & 0.93 & 0.88 \\
\hline 430 & 0.76 & 0.42 & 680 & 0.84 & 0.83 \\
\hline 440 & 0.75 & 0.47 & 690 & 0.61 & 0.68 \\
\hline 450 & 0.75 & 0.49 & 700 & 0.44 & 0.39 \\
\hline 460 & 0.73 & 0.53 & 710 & 0.32 & 0.16 \\
\hline 470 & 0.69 & 0.55 & 720 & 0.24 & 0.08 \\
\hline 480 & 0.69 & 0.59 & 730 & 0.16 & 0.05 \\
\hline 490 & 0.70 & 0.60 & 740 & 0.09 & 0.05 \\
\hline 500 & 0.71 & 0.65 & 750 & 0.04 & 0.05 \\
\hline 510 & 0.72 & 0.70 & 760 & 0.03 & 0.07 \\
\hline 520 & 0.73 & 0.81 & 770 & 0.01 & 0.09 \\
\hline 530 & 0.76 & 0.82 & 780 & 0.01 & 0.17 \\
\hline 540 & 0.82 & 0.86 & 790 & 0.00 & 0.27 \\
\hline 550 & 0.87 & 0.89 & 800 & 0.00 & 0.35 \\
\hline
\end{tabular}

${ }^{2}$ From McCree (1972).

${ }^{y}$ Derived from phytochrome absorbances and photochemical cross sections of Sager et al. (1988).

${ }^{x}$ Tabular data are adapted from Sager and McFarlane (1997).

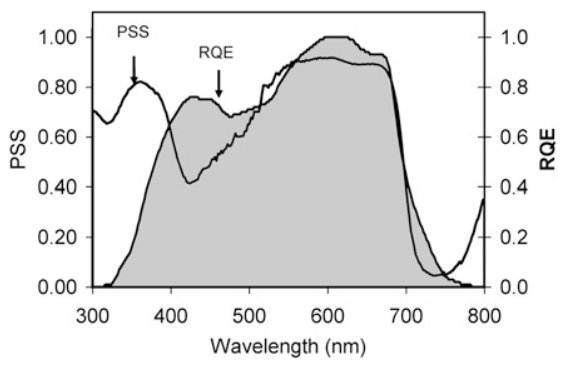

Fig. 4. Phytochrome photostationary state (PSS) and the relative quantum efficiency (RQE) of wavelengths ranging from 300 to $800 \mathrm{~nm}$. (Data derived from Sager and McFarlane, 1997.)

LEDs, by their nature, emit light of a wavelength from across the elemental junction and emit little or no radiant heat. However, there is a loss of heat from the diode junctions that must be accounted for. With high densities of high-output LEDs, the thermal output can be significant and must be managed to avoid damage to the LED array (Bourget, 2008).

Two examples are provided to illustrate the use of LEDs to regulate the phytochrome photostationary state to determine the effects of light quality of horticulturally significant characteristics. The first example is the effects of spectral quality on anthocyanin formation in red leaf lettuce when LEDs are the sole source of photosynthetic radiation. The second is the use of low-output red LEDs to alter the photostationary state of strawberry crowns before transplanting in high tunnels.

\section{SPECTRAL CONTROL OF ANTHOCYANIN FORMATION IN RED LEAF LETTUCE}

The induction of anthocyanins is known to be affected by light quality (Mancinelli et al., 1975). High concentrations of ultraviolet light have been associated with induction of anthocyanin (Krizek et al., 1998). Anthocyanin accumulation was observed in lettuce grown under high-output T-8 triphosphor fluorescent lamps (TPF; Sylvania FP541/ $841 /$ H0, Danvers, MA) with (Stutte et al., 2009) and without (Richards et al., 2004) acrylic barriers that blocked the transmission of ultraviolet light. Experiments were performed to determine if anthocyanin formation was under photoregulatory control, and if so, whether it was a phytochrome-mediated response. Red leaf lettuce cv. Outredgeous was grown under a broad-spectrum TPF lamp $(\Phi=0.66)$ and red $(660 \mathrm{~nm})$ plus far red (730 $\mathrm{nm})(\Phi=0.53)$ as controls because previous research had indicated that anthocyanin production was high under TPF (Richards et al., 2004; Stutte et al., 2009) and that the red/far red treatment had low anthocyanin content (Stutte et al., 2009). The threshold response for induction of phytochrome response is subject to some controversy (Franklin and Whitelam, 2005; Vandenbussche et al., 2005), but a general guide is that $\Phi>0.6$ is active.

Three LED treatments were selected with red $(660 \mathrm{~nm})$; red $(660 \mathrm{~nm})+$ blue $(440 \mathrm{~nm})$; and red $(660 \mathrm{~nm})$, green $(530 \mathrm{~nm})$, and blue $(440 \mathrm{~nm})$ that had identical $\Phi=0.72$ to 0.73 . In other words, $73 \%$ of the phytochrome was in the active $\left(\mathrm{P}_{\mathrm{fr}}\right)$ form (Stutte et al., 2009). The plants grown under TPF control had high anthocyanin content as expected. The lettuce grown under both red $(\Phi=0.73)$ and red/far red $(\Phi=0.53)$ failed to produce significant amounts of anthocyanin (Fig. 5). The addition of blue $(440 \mathrm{~nm})$ light significantly increased the concentration of anthocyanin in the leaf tissue as well as altered the developmental morphology of the lettuce, although there were no differences in either the total photosynthetically active radiation or $\Phi$ between the red $(\Phi=0.72)$ and red/blue $(\Phi=0.73)$ treatments (Fig. 5). The results shown in Figure 5 indicate that the presence of blue light, and not the phytochrome photostationary state, was critical in regulating the formation of anthocyanin. This suggests that the control of anthocyanin synthesis is associated with cryptochrome photoreceptors (Briggs and Huala, 1999) and is not a primary phytochrome-mediated developmental process.

Although the development of anthocyanin appears to be regulated by cryptochrome and not directly mediated by phytochrome, there were a number of developmental processes that were altered when $\Phi<0.60$. The addition of far red resulted in greater leaf elongation than red alone, but the epinastic response was less. This net effect was to increase the available leaf area for light interception over the other treatments (Stutte et al., 2009). The addition of far red decreased the total anthocyanin content in the leaf (data not shown) suggesting a possible secondary role for phytochrome.

\section{LIGHT-EMITTING DIODES TO UNDERSTAND THE FLOWERING RESPONSE OF A SHORT-DAY STRAWBERRY}

Takeda and Newell (2006) have observed that short-day strawberries that are grown in the greenhouse under long-day conditions can be induced to flower in the fall without exposure to cool temperatures or short days. This unexpected result was attributed to very high planting density (200 plant $\left./ \mathrm{m}^{2}\right)$ of the plug plants in the greenhouse. Takeda et al. (2008) stated that broad spectra light was absorbed by the canopy, but only wavelengths greater than $700 \mathrm{~nm}$ were being detected by the crown, resulting in an $\Phi<0.2$ at crown level. It was hypothesized that the early flowering response was phytochrome-mediated. To test this hypothesis, high-density strawberries were established under long days in a greenhouse $(\Phi=0.62)$ and then transferred to a controlled environment chamber with broad spectra fluorescent lamps $(\Phi=$ $0.66)$ under long-day conditions (16-h light/ 8-h dark). A strand of low-output red LEDs $\left(\lambda_{\max }=662\right)$ was used to illuminate the crown and increase the $\Phi$ to 0.75 (Fig. 6). This treatment was applied for $28 \mathrm{~d}$ and then the plants were transplanted in the field under a high tunnel production system.

After 2 months under high tunnel conditions, $83 \%$ of the plants without the supplemental red LED treatment were flowering, whereas less than half $(47 \%)$ of the plants with the LED treatment were producing flowers. These data strongly suggested the 


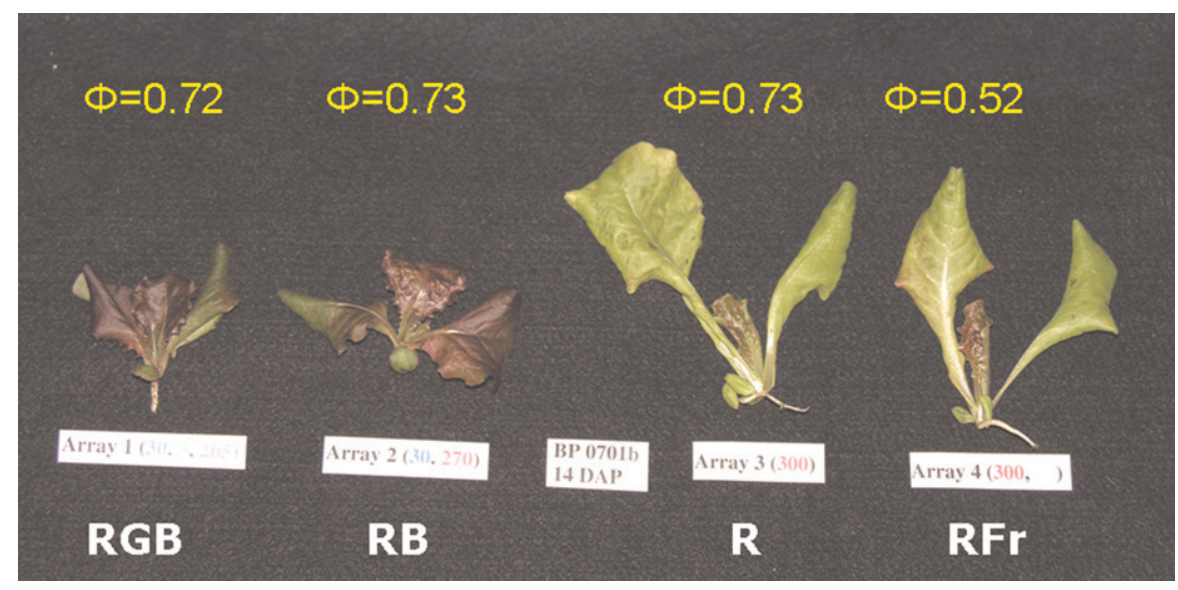

Fig. 5. Effect of spectral quality on the growth and anthocyanin formation of 14-d-old red leaf lettuce cv. Outredgeous grown under high-output light-emitting diodes at $300 \mu \mathrm{mol} \cdot \mathrm{m}^{2} \cdot \mathrm{s}^{-1}$. The treatments are expressed at RGB (red/green/blue); RB (red/blue); R (red); and RFr (red/far red) and the photostationary state $(\Phi)$ is provided. (Data derived from Stutte et al., 2009.)

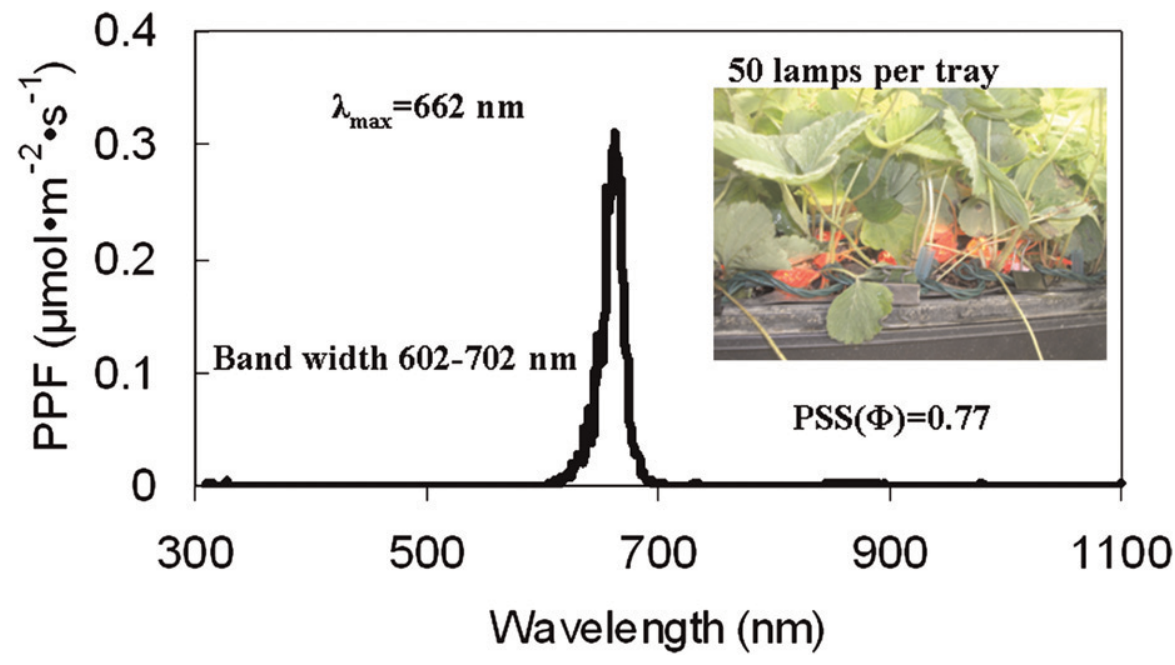

Fig. 6. Spectral distribution of low-output red light-emitting diodes used to alter the phytochrome photostationary state $(\Phi)$ of high-density strawberry plants before transplanting in high tunnels (inset). (Data derived from Takeda et al., 2008.)

maintenance of the vegetative state of the crown under short-day plants is under phytochrome control and suggests that manipulation of the crown light environment using LEDs to promote earlier flowering could be a tool to increase off-season production of strawberry.

\section{CONCLUSIONS}

Light-emitting diodes are durable, longlived, and versatile sources for plant lighting that can be used for plant growth. The selection of appropriate wavelengths of LEDs for a lighting source will allow customization of the spectrum to optimize growth quality and morphogenic responses. This may be through enhanced photosynthesis or photomorphogenesis. The rapid development of LEDs and corresponding commercial availability of affordable high-output LEDs provide the opportunity to develop the unique characteristics of LEDs as tools for research and commercial horticulture to enhance yield, extend seasons, and improve quality. thesis, and seed yield of wheat plants grown under red light-emitting diodes (LEDs) with and without supplemental blue lighting. J. Expt. Bot. 48:1407-1413.

Kim, H.H., R.M. Wheeler, J.C. Sager, N.C. Yorio, and G.D. Goins. 2005. Light-emitting diodes as an illumination source for plants: A review of research at Kennedy Space Center. Habitation (Elmsford) 10:71-78

Krizek, D.T., S.J. Britz, and R.M. Mirecki. 1998. Inhibitory effects of ambient levels of solar UV-A and UV-B radiation on growth of cV. New Red Fire Lettuce. Physiol. Plant. 103:1-7.

Mancinelli, A.L., C.-P.H. Yang, P. Lindquist, and O.R. Anderson. 1975. Photocontrol of anthocyanin synthesis III. The action of streptomycin on the synthesis of chlorophyll and anthocyanin. Plant Physiol. 55:251-257.

Massa, G.D., H.H. Kim, R.M. Wheeler, and C.A. Mitchell. 2008. Plant productivity in response to LED lighting. HortScience 43:1951-1956.

McCree, K.J. 1972. The action spectrum, absorbance and quantum yield of photosynthesis in crop plants. Agr. Meteorol. 9:191-216.

Morrow, R.C. 2008. LED lighting in horticulture. HortScience 43:1947-1950

Nagy, F. and E. Schafer. 2002. Phytochromes control photomorphogenesis by differentially regulated, interacting signaling pathways in higher plants. Annu. Rev. Plant Biol. 53:329-355

Paul, L.K. and J. Khurana. 2008. Phytochromemediated light signaling in plants: Emerging trends. Physiol. Molec. Biol. Plants. 14:9-22.

Pratt, L.H. 1982. Phytochrome: The protein moiety. Annu. Rev. Plant Physiol. 33:357-382.

Richards, J.T., N.C. Yorio, S.L. Edney, C.E. Yunker, and G.W. Stutte. 2004. Evaluating growth characteristics and total anthocyanin content in three cultivars of red romaine-type lettuce (Lactuca sativa L.) in response to three lighting intensities. Proc. Plant Growth Regul. Soc. Amer. 31:110-115.

Rockwell, N.C., Y.-S. Su, and J.C. Lagarias. 2006. Phytochrome structure and signaling mechanisms. Annu. Rev. Plant Biol. 57:837-858.

Sager, J.C. and J.C. McFarlane. 1997. Radiation, p. 1-29. In: Langhans, R.W. and T.W. Tibbitts (eds.). Plant growth chamber handbook. North Central Regional Research Publication No. 340. Iowa State Univ., Ames, IA

Sager, J.C., W.O. Smith, J.L. Edwards, and K.L. Cyr. 1988. The use of spectral data to determine photosynthetic efficiency and phytochrome photoequilibria. Trans. Amer. Soc. Agr. Eng. 31:1882-1889.

Salisbury, F.B. and C. Ross. 1992. Plant physiology. 4th Ed. Wadsworth, Belmont, CA.

Smith, H. and G.C. Whitelam. 1990. Phytochrome a family of photoreceptors with multiple physiological roles. Plant Cell Environ. 13:695-707.

Stutte, G.W., S. Edney, and T. Skerritt. 2009. Photoregulation of bioprotectant content of red leaf lettuce with light emitting diodes. HortScience 44:79-82.

Takeda, F., D.M. Glenn, and G.W. Stutte. 2008 Red light affects flowering under long days in a short-day strawberry cultivar. HortScience 43:2245-2247.

Takeda, F. and M. Newell. 2006. A method for increasing fall flowering in short-day 'Carmine' strawberry. HortScience 39:480-481.

Vandenbussche, F., R. Pierik, F.F. Millenarr, L. Voesenek, and D. Van Der Straeten. 2005. Reaching out of the shade. Curr. Opin. Plant Biol. 8:462-485.

Wheeler, R.M. 2008. A historical background of plant lighting: An introduction to the workshop. HortScience 43:1942-1943. 